\title{
Inward-Turning Streamline-Traced Inlet Design Method for Low-Boom, Low-Drag Applications
}

\author{
Samuel E. Otto ${ }^{1}$ \\ Purdue University, West Lafayette, Indiana, 47906 \\ Charles J. Trefny ${ }^{2}$ and John W. Slater ${ }^{3}$ \\ NASA Glenn Research Center, Cleveland, Ohio, 44135
}

\begin{abstract}
A new design method for inward-turning, streamline-traced inlets is presented. Resulting designs are intended for low supersonic, low-drag, low-boom applications such as that required for NASA's proposed low-boom flight demonstration aircraft. A critical feature of these designs is the internal cowl lip angle that allows for little or no flow turning on the outer nacelle. Present methods using conical-flow "Busemann" parent flowfields have simply truncated, or otherwise modified the stream-traced contours to include this internal cowl angle. Such modifications disrupt the parent flowfield, reducing inlet performance and flow uniformity. The method presented herein merges a conical flowfield that includes a leading shock with a truncated Busemann flowfield in a manner that minimizes unwanted interactions. A leading internal cowl angle is now inherent in the parent flowfield, and inlet contours traced from this flowfield retain its high performance and good flow uniformity. CFD analysis of a candidate inlet design is presented that verifies the design technique, and reveals a "starting" issue with the basic geometry. A minor modification to the cowl lip region is shown to eliminate this phenomenon, thereby allowing starting and smooth transition to sub-critical operation as back-pressure is increased. An inlet critical-point total pressure recovery of $96 \%$ is achieved based on CFD results for a Mach 1.7 freestream design. Correction for boundary-layer displacement thickness, and sizing for a given engine airflow requirement are also discussed.
\end{abstract}

\footnotetext{
${ }^{1}$ Intern, Inlets and Nozzles Branch, Purdue University, West Lafayette, IN, AIAA Student Member

${ }^{2}$ Aerospace Engineer, Inlets and Nozzles Branch, 21000 Brookpark Rd. Mail Stop 5-11, NASA Glenn Research Center, AIAA Senior Member

${ }^{3}$ Aerospace Engineer, Inlets and Nozzles Branch, 21000 Brookpark Rd. Mail Stop 5-12, NASA Glenn Research Center, AIAA Senior Member
} 


\section{Nomenclature}

$\begin{array}{ll}\text { A } & =\text { cross-sectional area } \\ L & =\text { length } \\ M & =\text { Mach number } \\ P & =\text { pressure } \\ r & =\text { radius } \\ \delta & =\text { flow deflection angle } \\ v & =\text { Prandtl-Meyer function } \\ \theta & =\text { conical flow azimuthal angle }\end{array}$

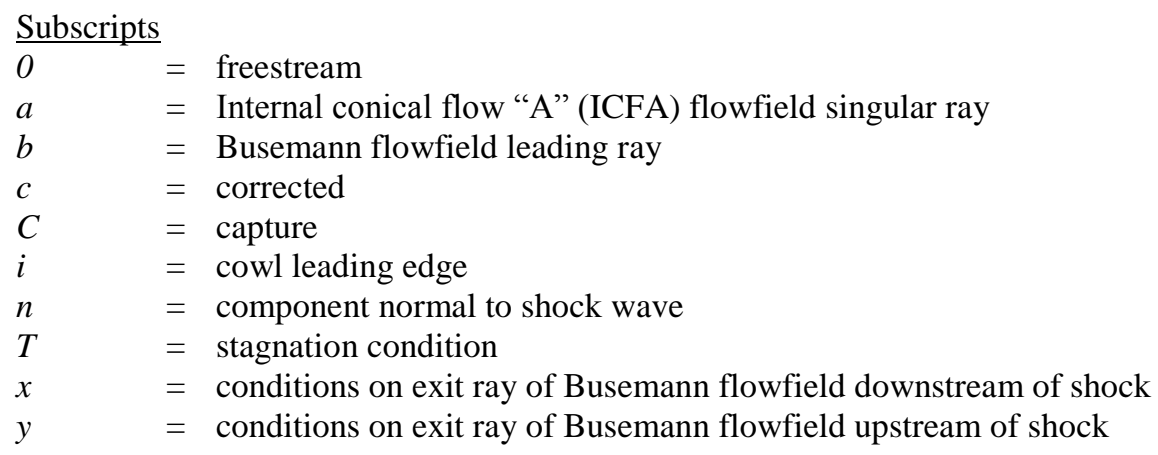

\section{Introduction}

Development of commercial supersonic flight has been hindered by many related factors including fuel-efficiency, economics, and sonic-boom signatures that have prevented over-land flight. Materials, propulsion, and flight control technologies have developed to the point where, if over-land flight were permissible, a commercial supersonic transport could be economically viable. Interest in over-land supersonic flight therefore forms the basis of a National Aeronautics and Space Administration (NASA) program to reduce sonic boom to acceptable levels. This research is led by the Commercial Supersonic Technology Project under NASA's Advanced Air Vehicles Program in cooperation with a number of U.S. aircraft manufacturers.

Computational fluid dynamics and modern optimization techniques enable designers to reduce the boom signature of candidate aircraft configurations to acceptable levels. However, propulsion systems must be carefully integrated with these low-boom configurations in order that the overall signatures remain acceptable. A significant contributor to the propulsion system's drag and sonic boom is the supersonic inlet. One approach is to mount the inlet above the wing, such that the wing provides shielding from the shock waves generated by the inlet and nacelle. This top-mounted approach introduces a number of issues with inlet design and performance however. The highly-swept wing configurations common to low-boom designs lead to non-uniform flow approaching the inlet, complicating the design of inlet compression surfaces. Also, the local Mach number is higher than the flight Mach number by up to one-tenth, which results in an increase in the required inlet capture area and a reduction in total pressure recovery. Another solution is to reduce the strength of waves generated by the nacelle to enable under-wing integration. The advantages of this approach include reduced inlet size and weight, and increased performance.

External-compression inlet designs are commonly used for the low supersonic speed regime being considered herein. This class of inlet generally employs an outward-turning shock and isentropic compression system located entirely forward of the cowl lip. The resulting inlet configurations exhibit stable sub-critical operation over a wide range of mass flow ratios since subsonic spillage around the cowl is allowed inherently by the design. However, the flow must be turned back to the propulsion system axis by the cowl which results in forward-facing nacelle area with concomitant drag and sonic boom. The increase in required turning angle with flight Mach number limits the applicability of external compression designs to the low supersonic regime. An inward-turning compressive flowfield can eliminate the external cowl angle responsible for the drag and sonic boom as well as offer the potential for reduced length and weight. However, inward-turning flowfields are necessarily formed downstream of the cowl lip and must ingest the compressive shock structure in a process known as "starting" before they can operate on-design. Furthermore, internal-compression generally prevents a gradual transition to sub-critical operation since the internal shock structure must be expelled, resulting in an abrupt loss of inlet performance known as "unstart." 
Streamline tracing was proposed by Molder ${ }^{1}$ as a means to overcome the starting issue for inward-turning hypersonic inlets based on the Busemann ${ }^{2}$ parent flowfield shown in Fig. 1. The axisymmetric Busemann flowfield is "conical" whereby conditions are constant along rays emanating from a focal point on the axis. The isentropic compression terminates with a conical shock wave coincident with the last ray. A drawback to the isentropic Busemann compression surface is its length, especially for high Mach number inlets, extending forward until parallel to the freestream. Also, the lack of an initial inward deflection places all required structural thickness to be outside the nacelle, and defeats the purpose of using an inward-turning flowfield to reduce sonic boom and drag. Techniques for truncation of Busemann flowfields for hypersonic applications have been reported $^{3-8}$ but all introduce significant nonuniformity in the outflow.

An inward-turning Mach 2.35 design that bears mention is the "parametric" inlet". It was intended to exploit the operational advantage of external compression to higher flight Mach numbers by reducing the extent of the cowl lip and thereby its drag. Supersonic compression was accomplished in an annular sector by an inwardturning outer ramp based on conical compression. A variable throat was required however, and the radial sidewalls of the sector were not streamlinetraced, but shaped to accommodate the pivoting compression ramp. This, along with supersonic corner flows and transition from sector to circular cross-section in the subsonic diffuser led to

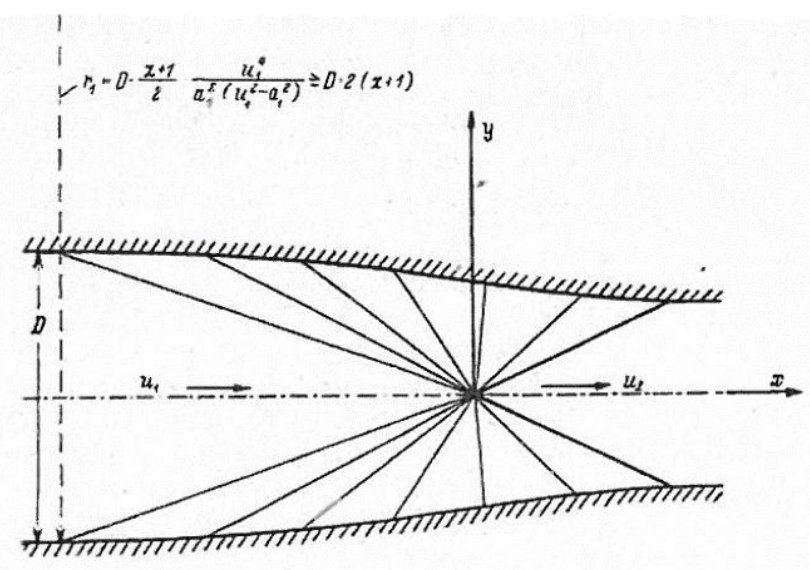

Bilı 5. Yerdichtungselüse für die Maclıselec Zúll $M d u=\frac{u_{1}}{a_{2}}=2$

Figure 1. Busemann conical compression. Reproduced from Ref 2 . relatively high flow non-uniformity.

Slater ${ }^{10}$ presented a low supersonic Mach number inward-turning inlet design based on a truncated Busemann flowfield, but as in the hypersonic case, conditions downstream of the leading shock were not well-matched to the conical compression, resulting in flow non-uniformity and unacceptable distortion at the engine face. Slater also compared the wave drag of his "STEX" inlets to that of axisymmetric spike, two-dimensional, and pitot inlet configurations. He reported nearly an order of magnitude reduction in nacelle wave drag for the inward-turning designs. While nacelle drag is important in its own right to the aircraft designer, the attendant reduction in the inlet's contribution to sonic boom is also key to the viability of commercial over-land supersonic flight.

Given the aforementioned advantages of inward-turning inlets, the present work extends that of Slater and presents a strategy for merging a leading conical shock wave with the Busemann flowfield in a manner that minimizes flow non-uniformity, especially near the axis of symmetry. Various merging techniques were evaluated with axisymmetric, inviscid (Euler) computational fluid dynamic (CFD) calculations leading to the discovery of the present method. The resulting parent flowfield features an initial inward radial flow angle that provides for low sonic boom and drag, and retains the high efficiency of the isentropic conical compression. Streamline-tracing adjacent to the axis-of-symmetry resulted in a compact supersonic diffuser with a scarfed aperture to allow for starting and stable sub-critical operation. A Mach 1.7 inlet design was then developed including viscous corrections to the analytical contour, and a subsonic diffuser. Finally, performance and operability of the resulting configuration was evaluated using CFD methods solving the Reynolds-Averaged Navier-Stokes (RANS) equations over a range of corrected flows.

\section{Inlet Design}

The class of supersonic inlets considered here are formed by tracing a collection of streamlines through an efficient compressive parent flowfield. An arbitrary closed circuit of points is chosen at a downstream location in the parent flowfield and traced upstream to form the supersonic compression surface of the inlet. Streamlines are terminated where the freestream flow is encountered, forming the inlet aperture. Careful selection of this downstream tracing curve and the parent flowfield itself allow for improved airframe integration and aperture shaping. Proper aperture shaping may be used to enhance operability in terms of starting and stable sub-critical operation by allowing for flow spillage. A scarfed aperture with a recessed vent region is desirable and may be achieved by tracing through a focused compression field. The vent region of the inlet aperture is formed by choosing points of the tracing curve whose streamlines extend upstream and terminate in the neighborhood of the compression field's focal point. 
Once the supersonic compression surface is formed by the above streamline tracing technique, it is modified in order to account for and control boundary-layer growth. This includes locally displacing the supersonic compression surface by the displacement thickness of the boundary-layer, as well as rounding the shoulder and introducing porous bleed as necessary. A subsonic diffuser is then extended from the shoulder back to the aerodynamic interface plane (AIP) in order to supply flow to the engine at the correct subsonic Mach number.

\section{A. Parent Flowfield Architecture}

Features of the parent flowfield are directly reflected in the final inlet geometry as well as in its operational characteristics and performance. Hence, desired inlet properties may be achieved by properly integrating them into the design of the parent flowfield from which the inlet is formed. In order to attain high performance in terms of total pressure recovery and drag, the parent flowfield must be efficient and compressive in nature. An inward-turning flowfield incorporating a leading oblique shock wave is also required in order to minimize the external cowl angle which is responsible for the drag and boom characteristics of the inlet. Axisymmetric flow is also necessary for the formation of a scarfed aperture while minimizing the size of the cowl lip region. A trailing oblique shock wave is necessary to turn the flow parallel to the symmetry axis. The desired parent flowfield is therefore conically (or approximately conically) symmetric with leading and trailing conical shock waves separated by a region of isentropic compression.

The conical exit wave may be a weak or strong oblique shock solution, resulting in two different parent flowfield configurations. In the case of a weak conical exit shock, the flow remains supersonic in the throat, and for stability is expanded slightly to about Mach 1.3 before passing through a "terminal" normal shock wave. The Busemann exit Mach number is therefore set to roughly 1.2. This configuration is illustrated in Fig. 2. Even with a scarfed aperture, the terminal normal shock wave is entirely internal and therefore may result in starting issues, as well as, the possibility for unstart events. As back pressure is increased, the normal shock wave will move forward of the throat thus triggering an unstart. It may be possible to incorporate flow spillage doors near the cowl lip in order to facilitate starting of designs utilizing the weak exit shock wave.

The second configuration utilizes a strong exit shock wave solution and is shown in Fig. 3. Inlets traced through this two-shock architecture with a streamline on the rotational axis, will have improved starting characteristics due to the transition from supersonic to subsonic flow at the focal point. The resulting scarfed inlet aperture will allow for sub-critical spillage without significant change in shock structure or unstart. Additionally, inlet designs utilizing the strong exit shock wave solution will have reduced length and weight as compared to an equivalent design with a weak exit shock wave.

While the strong exit shock wave architecture is advantageous in terms of weight and operability, it may suffer from losses in performance at higher freestream Mach numbers. It will be shown later that there is a practical limit to the freestream Mach number for this architecture caused by increasing strength of the terminal shock. The weak exit shock wave architecture delays the onset of this

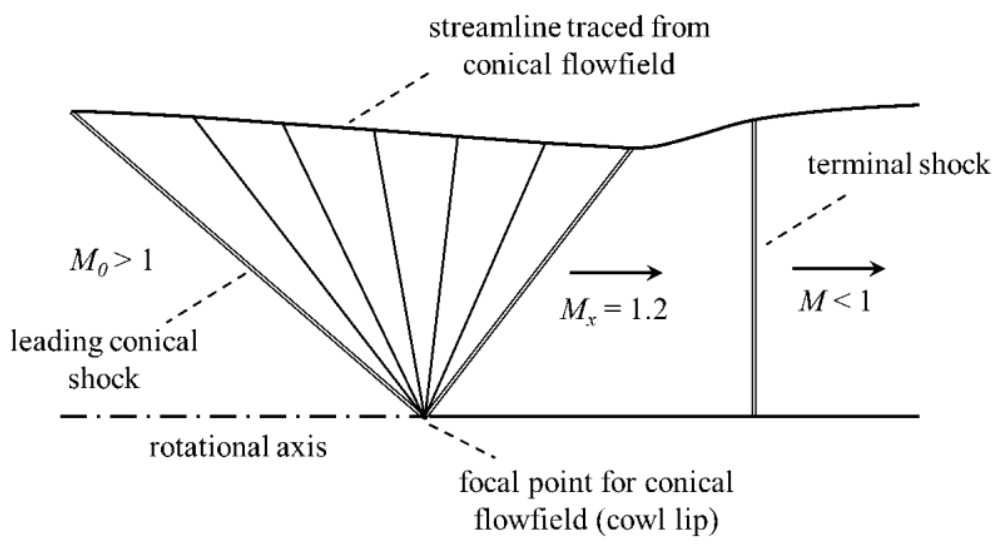

Figure 2. Weak exit shock wave configuration.

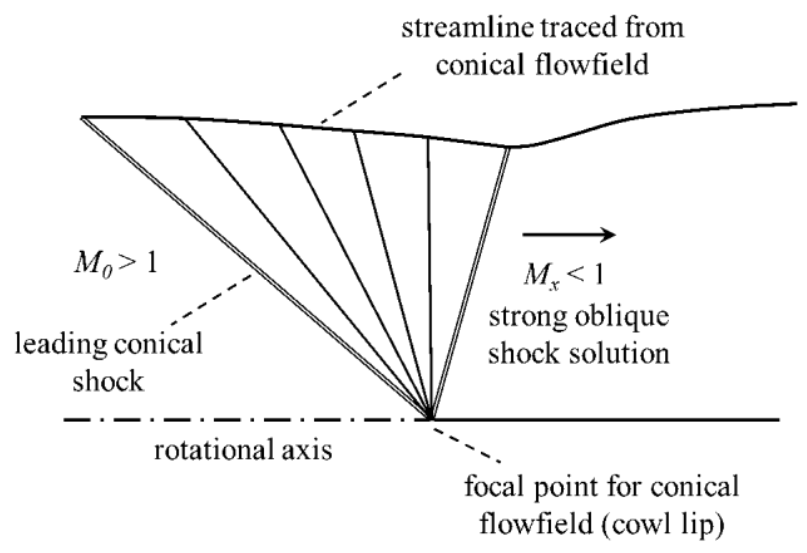

Figure 3. Strong exit shock wave configuration. 
problem substantially and may therefore be beneficial at higher flight Mach numbers. For the Mach 1.7 design being considered herein, the strong exit shock wave architecture is adopted.

It is desirable to utilize well-understood analytical flow solutions in the design of the parent flowfield in order to facilitate the design process. The conical Busemann flowfield is typically used to provide efficient, inward-turning compression. Conical flows are axisymmetric and isentropic, with constant properties along rays that emanate from a focal point on the rotational axis. They are governed by the well-known Taylor-Maccoll equations which relate the non-dimensional velocity components of the flow along a ray, to the angle of the ray from the rotational axis.

While being efficient, compressive, and inward-turning, the Busemann flowfield has a long upstream extent to the location where the flow is parallel with the axis. Truncation is typically used in order to reduce the overall length as well as to accommodate a finite internal cowl angle. In order to generate the truncated Busemann flowfield shown in Fig. 4, the Taylor-Maccoll equations are marched upstream in $\theta$ from the conical, oblique exit shock to the upstream truncation ray. The flow along the truncation ray has Mach number, $M_{b}$ and nonzero deflection angle, $\delta_{b}$. These upstream conditions at the truncation ray are determined completely by the exit shock wave angle, $\theta_{\mathrm{x}}$ and the outflow Mach number, $\mathbf{M}_{\mathrm{x}}$. Hence, in order to match specific upstream conditions at a fixed outflow Mach number, iteration must be performed on the exit shock wave angle.

While truncation is used to shorten the Busemann flowfield and accommodate a finite internal cowl angle, it cannot account for the attendant leading oblique shock wave. Attempts to design inlets based solely on truncated Busemann $\operatorname{march} \theta$ upstream

flowfields have met with only limited success due to flow non-uniformity caused by the leading shock wave ${ }^{3-5}$. This is because the isentropic compression upstream of the truncation point cannot be replaced by a shock wave. The shock wave formed at the leading edge of truncated Busemann inlets has a shallower angle than the truncation ray itself, extending into the region of conical flow. Impinging isentropic compression waves cause the leading shock wave to become curved, gaining strength, near the axis. This results in reduced total pressure recovery and increased flow distortion.

\section{B. Internal Conical Flow "A"}

In order to retain the high performance of the Busemann flowfield with truncation, a leading conical shock wave must be properly incorporated into the parent flowfield. This may be accomplished by introducing another conical flow solution called Internal Conical Flow-A (ICFA) described in reference 11 and shown in Fig. 5. ICFA is a solution to the Taylor-Maccoll equations marching clockwise in $\theta$ from conditions downstream of a leading conical shock wave. In doing so, one encounters a numerical singularity beyond which conical flow, as described by the TaylorMaccoll equations, is impossible. This ray is termed the singular ray, and a streamline of the ICFA flowfield between the leading conical shock wave and the singular ray is called an ICFA contour. This contour may be used to produce an approximately conical shock wave. The conical nature of the real flowfield produced by an ICFA contour is confined to a smaller sub-region bounded by the leading shock wave and a limiting characteristic originating at the trailing edge of the ICFA contour. Downstream of the limiting characteristic, the ICFA contour no longer has exclusive influence over the flow. Moreover, there exists a point near the axis on the leading oblique wave where the limiting characteristic intersects. This point is called the Rylov point and marks the location where the leading wave is no longer conical. Between the Rylov point and the axis, the shock wave becomes curved and eventually forms a Mach disc. This is due to the impossibility of regular shock wave reflection in axisymmetric flow $^{12}$. The location of the Rylov point and the severity of the leading wave rounding are assessed from numerical simulation and were not factored into the design process of the parent flowfield.

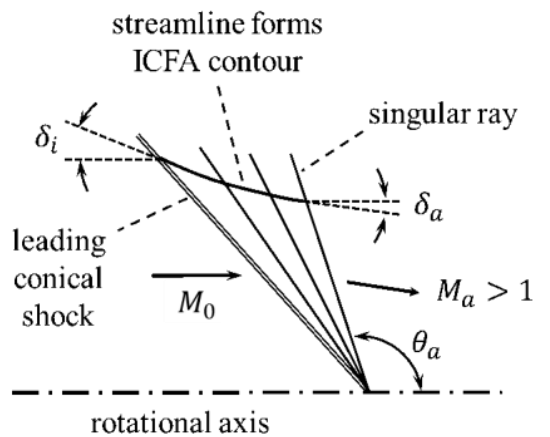

Figure 5. Internal Conical Flow-A (ICFA) flowfield. 
The ICFA and truncated Busemann flowfields must be merged in order to incorporate the leading conical shock wave into the parent flowfield. Ideally, the two flowfields could simply be connected by truncating the Busemann flowfield at the singular ray of the ICFA flow. However, the impossibility of conical flow downstream of the singular ray prevents this matching from being exact and leads to non-uniformity downstream in the conical compression. Ramasubramanian et al. ${ }^{7}$ explored matching various velocity components on the singular ray for a hypersonic application, and showed that the ICFA leading edge can be used to improve performance over simple truncation. However, effects on flow uniformity and distortion, important in the present context, were not considered. The next section presents the development of a new merging procedure for the low supersonic turbofan application, with the objective of improved pressure recovery, distortion, and inlet length.

\section{Merging Procedures}

In order to form a parent flowfield which minimizes unwanted interactions, the flow conditions along the truncation ray of the Busemann flowfield must closely resemble those at the ICFA outlet. The conditions on any ray of a conical flowfield are a unique function of the azimuthal angle, and are given by the non-dimensional radial and azimuthal velocity components or the Mach number and deflection angle of the flow. The design of the ICFA contour is entirely constrained by the freestream Mach number and choice of internal cowl angle. Hence, the angular location and flow conditions at the ICFA outlet are known immediately from the design constraints. This leaves three variables for matching along the truncation ray of the Busemann flowfield: the local Mach number, deflection angle, and the angle of the truncation ray itself. With the outflow Mach number of the entire compression process fixed by design, only the exit shock wave angle and truncation ray angle remain as free variables for merging the truncated Busemann and ICFA flowfields. In order to compare various merging strategies, the freestream Mach number, $M_{0}$, is constrained to 1.7 , the internal cowl angle, $\delta_{i}$, to $-5.0^{0}$, and the outflow Mach number, $M_{x}$, to 0.8 . These constraints are sufficient to uniquely specify the parent flowfield design using a given merging procedure.

Inviscid (Euler) CFD analysis was used to evaluate the merit of each merging procedure. Interactions arising from the mismatch in conditions between the ICFA singular ray and Busemann truncation ray were observed and compared. The inviscid parent flowfield produced by each candidate merging procedure was calculated using the Wind-US CFD code on an axisymmetric structured grid. The geometry in Fig. 6 consisted of the candidate streamline contour followed by a short, mildly diverging segment, a short cylindrical section, and a converging-diverging nozzle to force the strong exit shock wave solution. Of course, the final area ratio of the nozzle had to be tuned slightly to supply ideal back pressure for placement of the exit conical shock wave at or near the

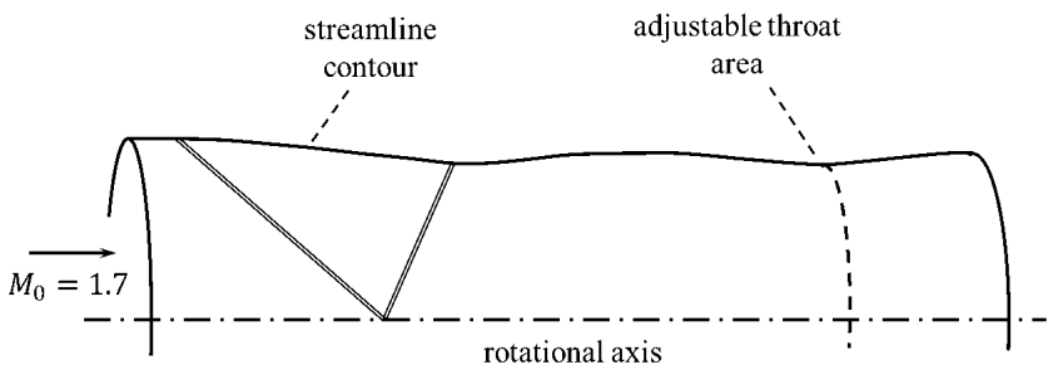

Figure 6. Axisymmetric Euler geometry. shoulder point.

The first merging procedure takes advantage of the fact that the truncation angle of the Busemann flowfield is a free variable. This allows for exact matching of the Mach number and flow deflection angle on the truncation ray to those of the ICFA outflow by iteration on the exit shock wave angle $\theta_{x}$. For each exit shock wave angle, the TaylorMaccoll equations are marched upstream until the ICFA flow deflection angle is matched. This forms the truncated Busemann flowfield which extends upstream to a location short of the ICFA singular ray. If the Mach number on this ray does not match that of the ICFA outflow, the exit shock wave angle is changed. The resulting parent flowfield contains a sliver between the ICFA singular ray and the truncation ray of the Busemann flowfield which is not accounted for by either the ICFA or truncated Busemann flowfield. The ICFA and truncated Busemann streamline contours are simply connected by a straight segment at the common flow deflection angle which spans the inherent gap.

The streamline resulting from the first merging procedure is shown in Fig. 7. The figure illustrates the relative sizes of the ICFA contour (ending at ray "a"), the truncated Busemann contour (ending at ray "b"), and the straight adjoining section shown as a dotted line. The ICFA contour is quite small even in comparison to the adjoining segment 
which results from the inherent error in the ray matching locations. This raises concerns over the radial location of the Rylov point since the limiting characteristic at the ICFA outlet has little upstream distance to travel before reaching the leading conical shock wave. If the interactions below the Rylov point are too severe, downstream flow uniformity will be compromised. Analytical values for the Mach number and deflection angle along key rays found from this merging procedure are presented in the table of figure 7 . Note that the Mach number and deflection angle match along the merging rays (a) and (b), but the ray angles are different. Additionally, the area contraction ratio is insufficient for starting when compared to the Kantrowitz limit. However, subsonic flow spillage once streamline-tracing is performed may be sufficient to account for this difference.

Inviscid CFD results for the streamline profile generated using merging procedure 1 are shown Fig. 8. The leading oblique shock wave appears to be well-formed and follows the analytical shock position shown as a white dashed line. As expected, the shock wave begins to round and forms a Mach disc near the axis. These results indicate that the ICFA contour, while small, is effective at producing a nearly conical shock wave. Additionally, the location of the exit shock wave agrees approximately with that predicted by the merging procedure. However, imperfect application of back pressure resulted in the exit shock wave landing slightly behind the shoulder point. Expansion around the corner and a small normal shock wave produce the slip line observed along the upper surface. The straight adjoining section between the ICFA and Busemann contours causes a large region of non-uniform, non-conical flow which propagates towards the axis. The flow in this region also appears to drive the formation of a large bifurcated shock system near the axis. Two slip lines are formed as a result of the bifurcated exit shock wave and reduce flow uniformity downstream of the throat. Despite these interactions, a region of approximately conical flow is formed by the truncated Busemann contour and bounded downstream by the bifurcated exit shock wave. Additional compression provided by the straight section yields a slightly decreased Mach number, $M_{y} \cong 1.30$ ahead of the exit shock wave.

The second merging procedure is that adopted by You, et al. ${ }^{8}$ The Busemann flowfield truncation angle is constrained so that it matches the singular ray angle of the ICFA flowfield. Hence, the two flowfields are merged along the same ray and no straight connecting section is necessary. In this merging procedure, the Busemann exit shock wave angle is varied until the Mach number along the truncation ray of the Busemann flowfield matches the outflow Mach number of the ICFA flowfield. Although the interface Mach numbers match, the flow deflection angles do not. According to the results in reference 12 for a Mach 6 design, the flow deflection angle mismatch causes a supersonic expansion. This results in off-design operation of the truncated Busemann section of the inlet and improper 
formation of the compressive flow structures. Rather than forming a single Busemann exit shock wave, two waves form where the expansion fan reaches the rotational axis. Being that the Busemann section of the inlet is operating super-critically, both of these waves land behind the inlet shoulder, causing flow distortion.

The streamline generated using the second merging procedure is shown in Fig. 9. In this figure, locations (a) and (b) correspond to conditions upstream and downstream of the merging ray. From the table, the angular mismatch at the merging ray (shown in bold) indicates that the flow will be expanded around a $2.212^{\circ}$ corner before entering the contour of the truncated Busemann flowfield. Such an expansion will cause the Mach number downstream of the merging ray, $M_{b}$ to increase to 1.656, significantly higher than the 1.581 inflow design value for the conical compression.

Inviscid CFD results for the streamline profile generated using procedure 2 are shown Fig. 10. The leading conical shock wave shows reduced rounding near the axis and formation of a smaller Mach disc when compared to the solution for merging procedure 1 shown in Fig. 8. Therefore, below the Rylov point, shaping of the leading conical shock wave is significantly influenced by the downstream flow. Effects of the deflection angle mismatch are evident in the formation of a pronounced expansion fan at the interface of the two contours. The expansion fan propagates downstream towards the axis and causes a bifurcated exit shock wave structure to form. The size of the bifurcated shock region and the Mach discs themselves are smaller than those in Fig. 8, indicating an improvement in overall flowfield uniformity. The accelerated flow downstream of the expansion fan causes the lines of iso-Mach number to become slightly curved in the truncated Busemann flowfield. Additionally, this acceleration causes the exit shock wave to be formed downstream of its design location and at a higher Mach number $M_{y} \cong 1.40$. Impinging Mach waves on the exit shock wave cause it to become somewhat curved as well, reducing downstream flow uniformity.

The third merging procedure attempts to correct for the flow expansion present in procedure 2. While the angular mismatch will always be present, it may be possible to account for its effect on the flowfield. Rather than matching the Mach numbers at the interface directly, the truncated Busemann flowfield is matched to the expanded Mach number through the deflection angle difference. As with procedure two, the Busemann flowfield is truncated at the singular ray of the ICFA flowfield. Iteration is performed on the exit shock wave angle so that the post-expansion Mach number is matched by the truncation ray of the Busemann flowfield:

$$
v\left(M_{b}\right)=v\left(M_{a}\right)+\left(\delta_{b}-\delta_{a}\right)
$$


This modification should allow the truncated Busemann section of the inlet contour to operate closer to its design Mach number. Accounting for the expansion fan in this way should improve the formation of the compressive flow structures, in particular the angle, location, and strength of the Busemann exit shock wave.

The streamline generated using the third procedure is shown in Fig. 11. The difference in Mach number upstream and downstream of the merging ray (stations "a" and "b") correspond to a Prandtl-Meyer expansion through the difference in deflection angles. Since the expansion fan is taken into account, additional contraction is needed to decelerate the flow to the correct exit conditions. Hence the throat area is necessarily smaller than that found using procedure 2 .

Inviscid CFD results for the streamline profile generated using procedure 3 are shown Fig. 12. The leading conical shock wave has the same shape as that in Fig. 11, showing minimal rounding and excellent agreement with the predicted geometry. The expansion fan still propagates towards the axis and triggers the formation of a bifurcated shock wave. However, the effect is significantly reduced and the size of the bifurcated shock wave region is much smaller than in Figs. 8 or 10. Additionally, the truncated Busemann compression field downstream of the expansion fan is well-formed with nearly straight lines of iso-Mach number impinging on the intended focal point. The exit shock wave is in much better agreement with the desired position predicted by the merging procedure. Proper formation of the conical compression field yields a uniform upstream Mach number, $M_{y}$ of 1.34 across the exit shock wave. This is in close agreement with the expected value given by the table in Fig. 11. Furthermore, interactions between the isentropic compression field and the exit shock wave are minimal, decreasing rounding of the shock wave and contributing to improved flow uniformity downstream. On these grounds, we conclude that the third merging procedure offers significant improvement over the first two and will be selected for our application.

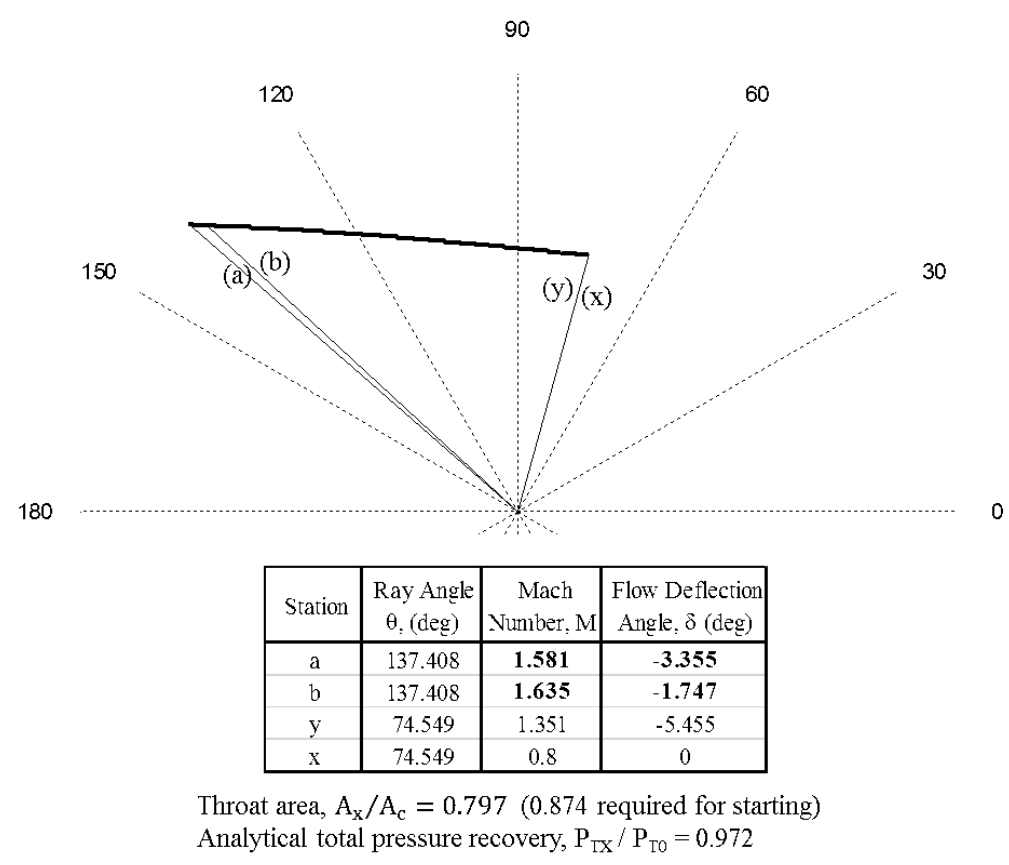

Figure 11. Analytical results for merging procedure 3.

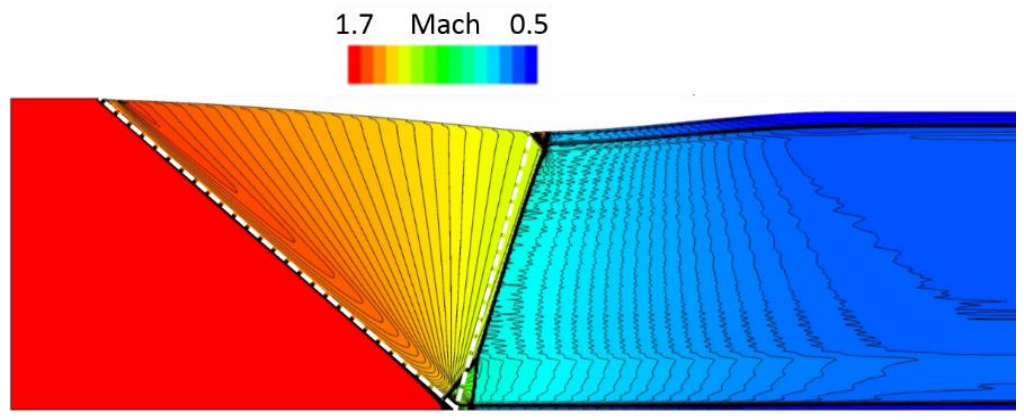

Figure 12. Inviscid flowfield of merging procedure 3. 


\section{Design Space for Merging Procedure 3}

The design space for parent flowfields generated using the third merging procedure and strong exit shock wave solution was mapped. Effects of freestream Mach number, internal cowl angle, and outflow Mach number design choices on three main performance metrics were determined using the analytical technique described above. The first performance metric considered is the total pressure recovery of the inlet design and is directly related to the useful work which may be extracted from the flow for the purpose of generating thrust. The second performance metric is the overall length of the supersonic compression surface which influences the final weight of the streamline traced design. Lastly, the normal component of the exit shock wave Mach number is considered. A value of 1.3 or below is typically desired to minimize shock boundary-layer interaction and flow separation in the subsonic diffuser. The variation of these parameters over the design space will be used to illustrate relevant physics in the parent flowfield, establish practical limitations, and drive selection of a final parent flowfield design.

Figure 13 shows the effect of outflow Mach number on total pressure recovery and the normal component of the exit shock wave Mach number at various levels of freestream Mach number. As the outflow Mach number is increased, the strength of the exit shock wave is reduced and thus the total pressure recovery is increased. Choosing a design outflow Mach number of 0.9 rather than 0.8 results in a significant reduction in the normal component of the exit shock wave Mach number, bringing it well below the threshold of 1.3 for the Mach 1.7 freestream case. Additionally, a nearly $2 \%$ increase in total pressure recovery is obtained. However, it should be noted that a higher outflow Mach number contributes to higher area ratio and increased length in the subsonic diffuser. Despite this, an outflow Mach number of 0.9 was chosen for the final parent flowfield design.

The freestream Mach number was fixed at 1.7 for the current inlet design; however its effect is interesting for future applications. As freestream Mach number increases, the strength of both the leading and exit conical shock waves are increased. Figure 13 shows that for constant outflow Mach number, the normal component of the exit shock wave Mach number increases with freestream Mach number. For an outflow Mach number of 0.9 , the 1.3 threshold for normal Mach number would be reached at approximately a freestream Mach number of 2.1. Above this freestream Mach number, it may become difficult to control boundary-layer separation at the shoulder. Another effect of increased freestream Mach number is increasing length. This is a well-known phenomenon in the design of Busemann inlets as more isentropic compression and thus upstream extent is needed to achieve the desired exit conditions.

The effects of varying leading edge internal cowl angle, $\delta_{i}$ and freestream Mach number, $M_{0}$ on inlet length are shown in Fig. 14. As the internal cowl angle, is increased, the inlet length and total pressure recovery are both reduced.

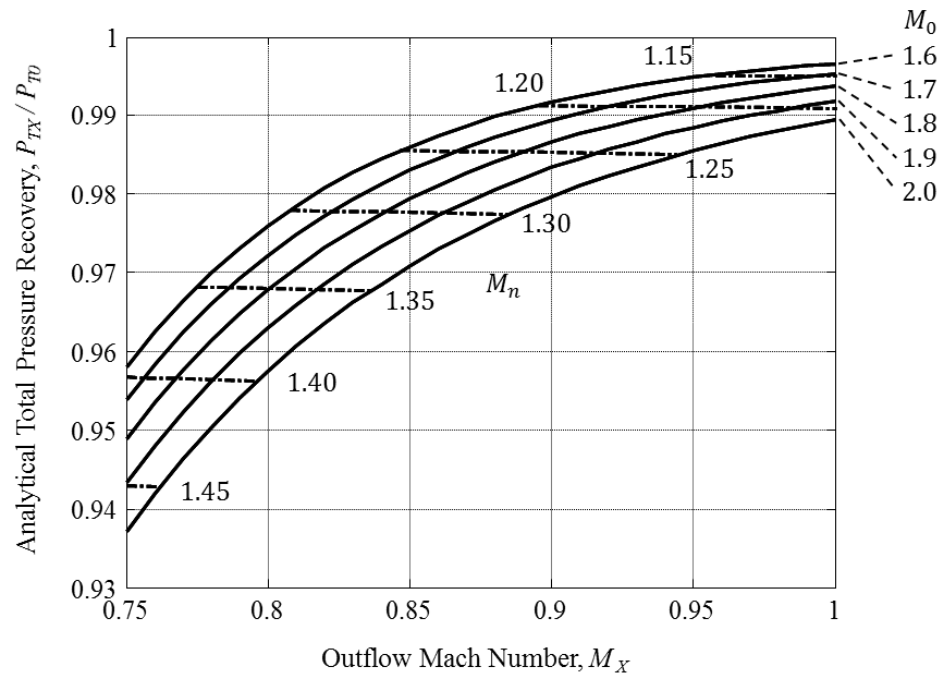

Figure 13. Total pressure recovery versus outflow Mach number for an internal cowl angle of $\mathbf{- 5 . 0 ^ { \circ }}$.

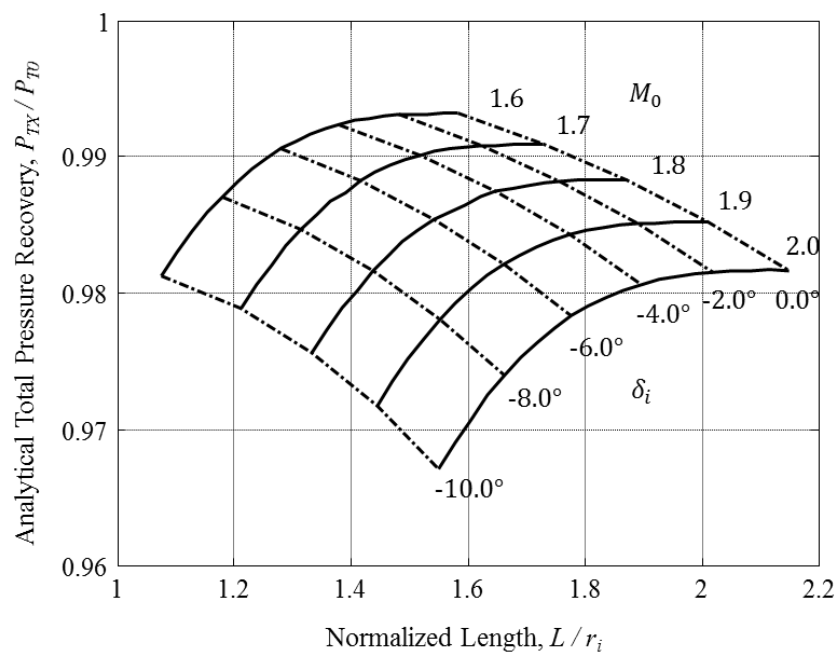

Figure 14. Total pressure recovery versus normalized inlet length for an exit Mach number of 0.9 . 
With more compression taking place through the leading shock wave, less isentropic compression is needed, shortening the region of conical flow. However, a stronger leading shock wave also causes greater losses in total pressure. For internal cowl angles between $0^{0}$ and $-5^{0}$, the length may be reduced considerably with minimal loss in total pressure recovery. Therefore, a leading edge internal cowl angle of $-5^{0}$ is a good design choice over the range of flight Mach numbers examined.

The streamline generated for the final design of the parent flowfield is shown in Fig. 15 along with values of the Mach number and deflection angle at key locations in the flow. Comparing the table to that in Fig. 11 shows that increasing the outflow Mach number from 0.8 to 0.9 increases the recovery and reduces the terminal shock Mach number as expected. The additional flow turning however, has the undesirable effect of reducing the throat area which can lead to concerns over starting.

Inviscid CFD results for the final streamline contour are shown in Fig. 16. The flowfield demonstrates the same advantageous features as the flowfield in Fig. 12 which was generated using the same merging procedure. Accurate formation of the Busemann flowfield is observed with straight, focused lines of iso-Mach number. The flow ahead of the exit shock wave is uniform at the expected Mach number of 1.27, contributing to its near exact agreement with the analytical shock position calculated in the merging procedure. While the shock wave is still bifurcated near the axis, the size of the bifurcated region is very small and produces a weaker slip line than the case in Fig. 12. Near ideal formation of the conical flowfield and exit shock wave produce highly uniform flow at a Mach number 0.91 in the throat. The mass-averaged total pressure recovery at the exit plane is 0.989 which matches the analytical solution to a somewhat surprising degree, indicating negligible additional shock losses due to merging. This makes the flowfield an excellent candidate for streamline tracing.

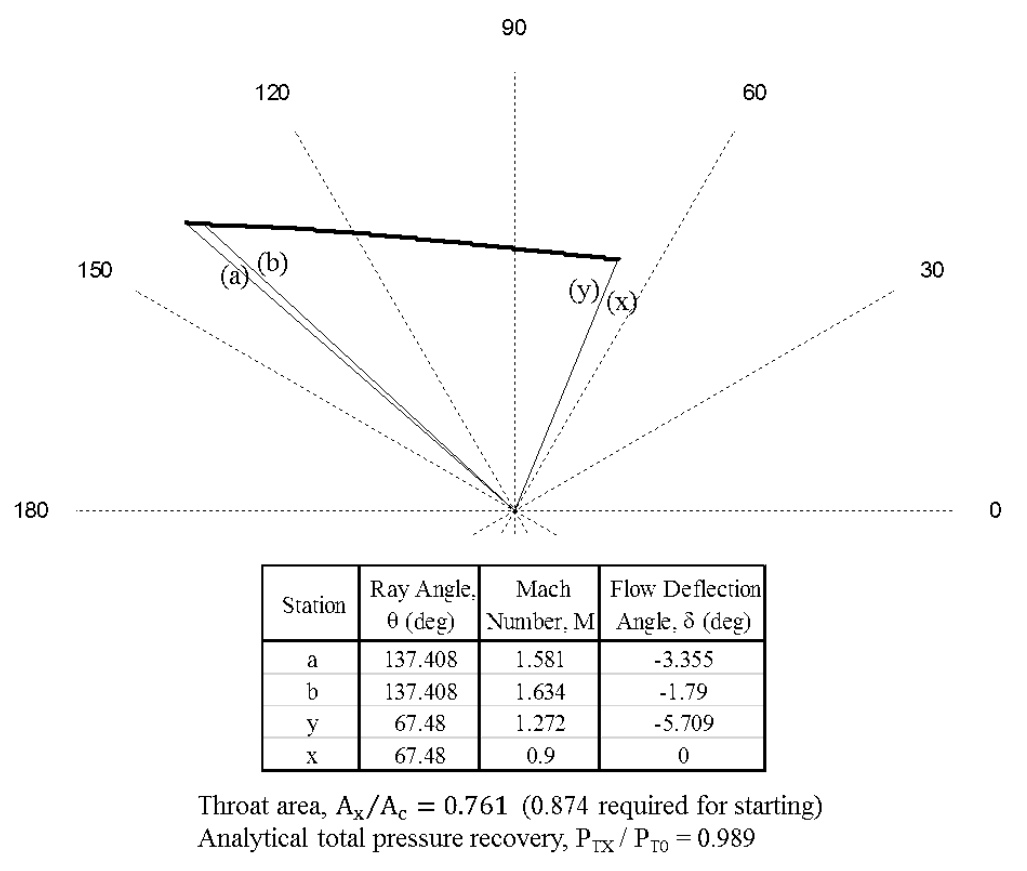

Figure 15. Analytical results for the final parent flowfield with an exit Mach number of 0.9 .

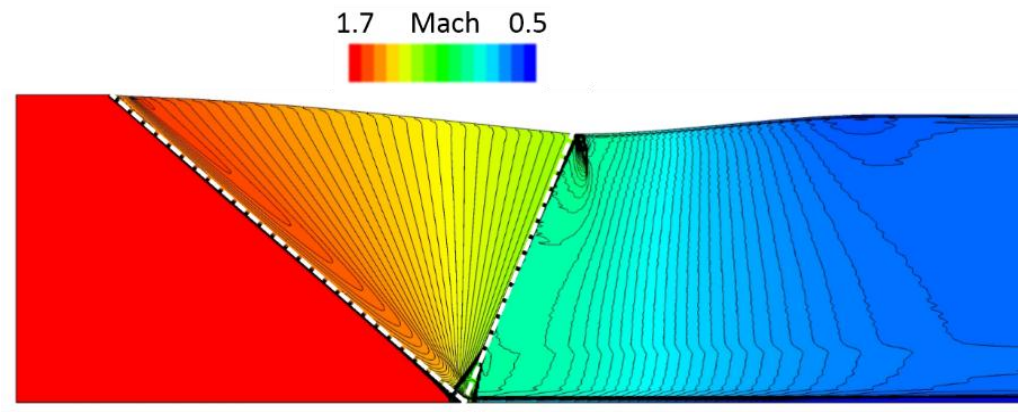

Figure 16. Inviscid flowfield for the final design with an exit Mach number of 0.9 .

\section{E. Development of Streamline-Traced Inlet Geometry}

A realistic inlet geometry is now developed, based on the final parent flowfield. Streamline tracing with a circular downstream tracing curve was used to form the supersonic compression surface of the inlet. The tracing curve was normal to the rotational axis of the Busemann parent flowfield and located axially at the end of the supersonic contour. The bottom of the tracing curve was placed on the axis. Thus the streamline on the rotational axis extends forward to the focal point of the parent flowfield, downstream of which the flow is subsonic. This is intended to provide a 
"venting" point for flow spillage and stable transition to subcritical operation. Streamline tracing results in the geometry pictured in Fig. 17.

A subsonic diffuser was generated using a concave, constant-pressure-gradient profile between the inlet throat and compressor face with an equivalent conical angle of $3^{0}$. Segments at the upstream and downstream ends were replaced by circular arcs such that both ends were parallel to the axis. This reduced the equivalent conical angle to $2.164^{\circ}$. At this point, the inlet was scaled based on the General Electric F404GE-102 low bypass ratio turbofan engine for a corrected airflow and inlet diameter of $146.3 \mathrm{lb} / \mathrm{sec}$ and 27.9-inches respectively. The total pressure recovery in the supersonic diffuser was taken to be $98.9 \%$ based on the design inviscid recovery. A recovery of $98 \%$ was assumed in the subsonic

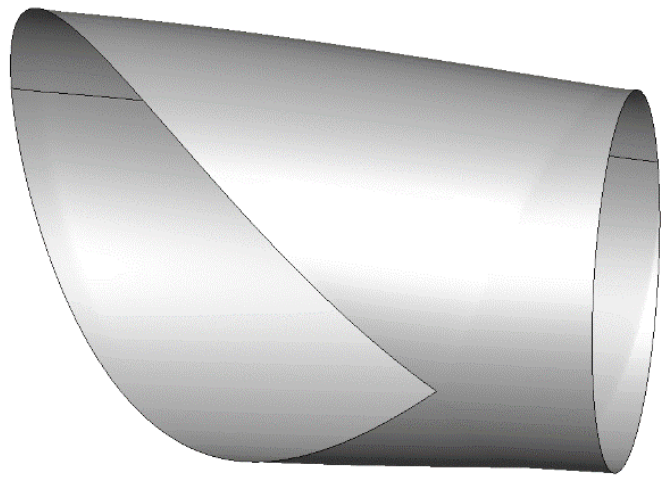

Figure 17. Streamline-traced inlet geometry. diffuser resulting in an overall recovery of $96.9 \%$, and an inlet capture area of $552.3 \mathrm{in}^{2}$.

Prior to grid generation and CFD analysis, experience suggested three modifications to the native geometry for improved performance and operability. The first modification is to round the corner at the impingement line of the exit shock wave or "shoulder" in order to avoid abruptly turning the boundary-layer and to accommodate any slight error in shock location. This is accomplished by replacing the 2-degree angular sector in the parent flowfield just upstream of the exit shock wave with a circular arc. The arc radius is sized to preserve the original throat area defined by the sharp corner, and varies circumferentially as the corner angle changes. This rounding should reduce the tendency of the boundary-layer to separate at the shock impingement point.

The second modification involved displacing the inlet compression surfaces outward to accommodate the boundary-layer displacement thickness thereby preserving the desired parent flowfield. Given the scarfed aperture of the streamline-traced design, the boundary-layer displacement thickness varies circumferentially at the tracing plane depending on distance from the cowl lip. Furthermore, the inward-turning nature of the supersonic compression surface along with the inherent adverse pressure gradient, results in thicker boundary layers than those of a flat plate. As an initial estimate, the tracing plane was displaced by three times that given by turbulent, incompressible flat-plate theory ${ }^{13}$. A "best-fit" circle was then used to define a new circular throat at the tracing plane. Along each streamline, the surface coordinates were then displaced in a direction normal to the local surface (not radial from the original axis) by an amount proportional to distance along the streamline to the $4 / 5$ power. The resulting modified supersonic compression surface was translated down such that the throat was concentric with the original throat at the tracing plane, and the subsonic diffuser was re-contoured to match the slightly larger throat diameter.

The last modification as shown in Fig. 18, was to increase the extent of the vent region at the focal point of the parent flowfield. The purpose of this change was to allow increased flow spillage during starting and sub-critical operation of the inlet. The vent region was modified by specifying a new downstream cowl lip location along with azimuthal angles to define the extent of the modification. The cowl was translated aft by an axial distance equal to $3 \%$ of the equivalent capture radius. The upstream half angle was set to $30^{\circ}$ and was used to specify the circumferential extent of the modification on the original cowl lip. The downstream half angle was set to $10^{\circ}$ and specified the extent of the new, un-swept cowl lip. A cubic polynomial was used in the space between the upstream and downstream angles in order to smoothly blend the recessed cowl

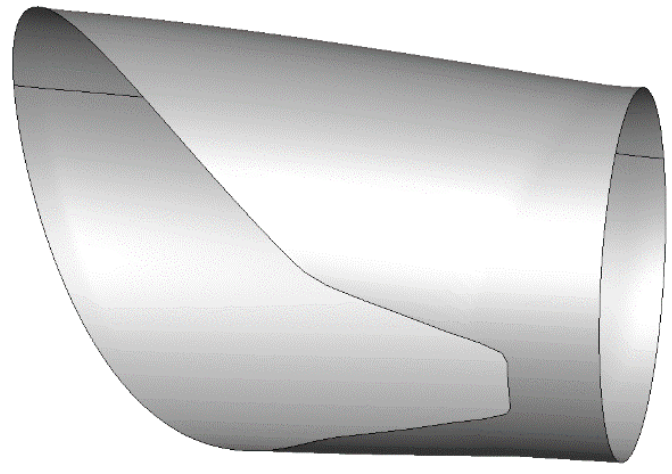

Figure 18. Modified inlet aperture and vent region. lip with the unmodified aperture. 


\section{Analysis Method}

CFD simulations were performed on the resulting inlet configuration using the Wind-US CFD code ${ }^{14}$. The WindUS CFD code solved the Reynolds-Averaged Navier-Stokes (RANS) equations for a multi-block, structured grid for a flow domain about and within the inlet. Fig. 19 shows the flow domain used for the CFD simulations of the inlet. The flow domain had inflow boundaries upstream and around the inlet where freestream boundary conditions were imposed. At the end of the cowl exterior, the domain had an outflow boundary where supersonic extrapolation boundary conditions were imposed. The internal and external surfaces of the inlet formed a portion of the boundary of the flow domain where adiabatic, no-slip viscous wall boundary conditions were imposed. Downstream of the engine face, a converging-diverging nozzle section was added to the flow domain to set the flow rate through the inlet. The nozzle throat was set to be choked so that the outflow boundary of the nozzle was supersonic, which allowed nonreflective extrapolation boundary conditions to be imposed.

The CFD grid consisted of 16 blocks and contained $3.476 \times 10^{6}$ grid points. The wall normal grid spacing was $1.0 \times 10^{-5}$ feet, which resulted in a normalized wall coordinate of $y^{+}<1.0$ throughout the inlet. Within the internal ducting of the inlet, the grid contained 321 axial grid points, 61 circumferential grid points, and 209 grid points between the bottom and

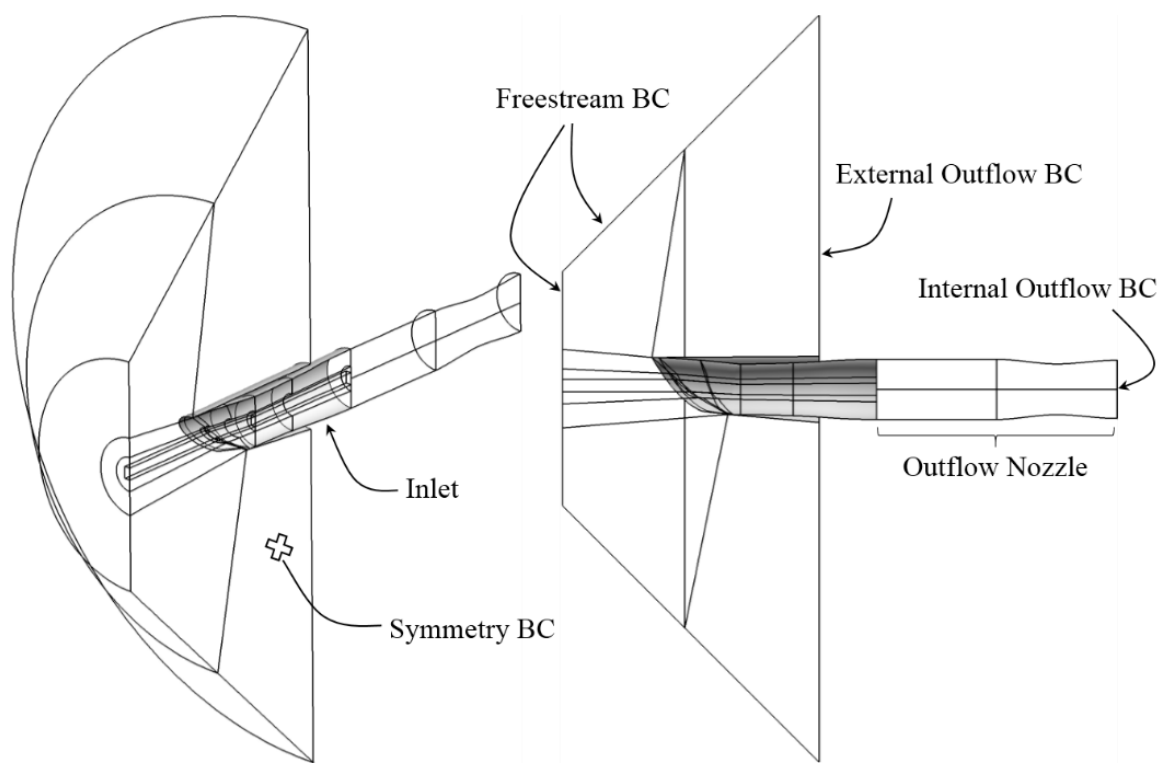

Figure 19. Computational domain and boundary conditions for the CFD analysis. top of the duct.

Wind-US solved the RANS equations in a time-dependent manner for the steady-state, turbulent, compressible flow using a cell-vertex, finite-volume, time-marching approach. A calorically perfect gas model was used. Turbulence was modeled using the two-equation Menter shear-stress transport (SST) model. Porous bleed was simulated as a boundary condition in which the bleed rate was allowed to vary according to local flow conditions as described in Ref. 15. The bleed flow was modeled as flowing into a plenum and then ejected out to the freestream through a choked nozzle with a fixed throat area. The primary inputs for the porous bleed model were the porosity of the bleed region and the area of the bleed exit nozzle. Figure 20 shows the boundary-layer bleed pattern used and its relationship to the design position of the exit shock wave. The bleed region varies in width from approximately 10 grid cells at $180^{\circ}$ to 25 cells at $0^{0}$ where the length of boundary-layer run is the greatest. Although depicted as discrete patches of grid cells, the bleed region was continuous in the circumferential direction.

The flowfield solution was initialized at all grid points with the freestream flow conditions. Spatial accuracy was formally second-order using the Roe flux-difference splitting upwind formulation. Steady-state flows were simulated through an iterative process using a first-order, implicit Euler method with local time-stepping. Iterative convergence of each solution was evaluated through monitoring of the convergence of the inlet flow rate, the total pressure recovery, and total pressure distortion. The steady-state solution was considered converged when these values varied by less than $0.1 \%$ of their values over hundreds of iterations. The solution

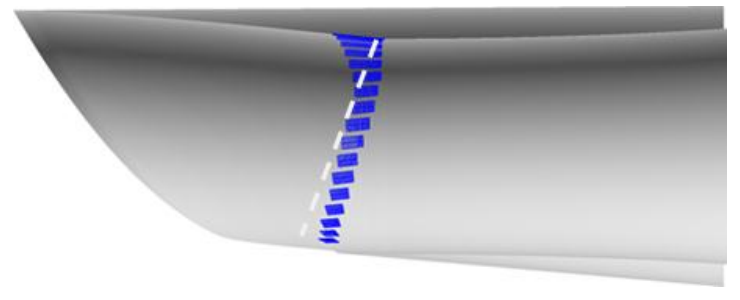

Figure 20. Boundary-layer bleed layout. 
residuals were also monitored to check that they reduced and approached steady values. Wind-US sequenced the grid to solve the flow on a coarser grid consisting of every other grid point. This accelerated the iterative convergence and created two solutions on two resolutions of grids. The total pressure recovery at the engine face between the two grid resolution levels differed by less than $1.0 \%$, which provided confidence that the grid sufficiently resolved the flow to provide the performance data for the inlet.

The CFD simulations were processed in several ways to obtain the inlet performance data. A CFD simulation was characterized by an outflow nozzle throat area ratio which was the cross-sectional area of the nozzle throat normalized by the area of the engine face. An integration of the flow through grid planes in the outflow nozzle block provided the inlet flow rate. The ratio of the inlet flow rate to the theoretical maximum capture flow rate provided the inlet mass flow ratio expressed as $A_{0} / A_{c}$. The integration of the flow through the porous bleed surface provided the bleed flow rate, which was then normalized by the capture flow rate. The total pressure recovery and distortion descriptors were obtained through interpolation of the CFD solution onto an equal-area 40-probe rake, positioned at the AIP, as specified by the SAE 1420 recommendations ${ }^{16}$. The total pressure recovery, $P_{T 2} / P_{T O}$ was calculated as the average of all of the total pressures of the probes normalized by the freestream total pressure. The inlet corrected flow rate $\left(W_{c}\right)$ was then calculated using the actual inlet flow rate and total pressure recovery. The total pressure distortion descriptors were the General Electric radial tip (IDR) and circumferential (IDC) descriptors calculated using the "Phase 0 Method D" methodology as described in reference 17.

\section{Results and Discussion}

Results of CFD simulations performed for the inlet over a range of corrected flows from supercritical to subcritical are now presented and discussed. The corrected flow was varied by changing the nozzle throat area. Nozzle throat area ratios and performance parameters are listed in table 1. Cases denoted "b" are at the critical point, "a" are super-

Table 1. Inlet performance and nozzle settings.

\begin{tabular}{|c|c|c|c|c|c|c|c|}
\hline $\begin{array}{l}\tilde{J} \\
\tilde{J}\end{array}$ & 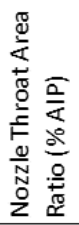 & 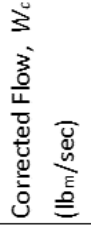 & 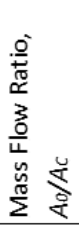 & 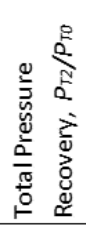 & 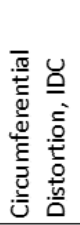 & 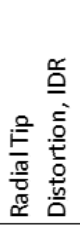 & 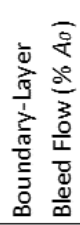 \\
\hline & $72.0 \%$ & 153.1 & 0.987 & 0.906 & 0.114 & 0.109 & $0.00 \%$ \\
\hline \multirow[t]{3}{*}{ a } & $71.5 \%$ & 152.0 & 0.987 & 0.914 & 0.104 & 0.108 & $0.00 \%$ \\
\hline & $71.3 \%$ & 151.3 & 0.987 & 0.920 & 0.097 & 0.106 & $0.00 \%$ \\
\hline & $71.2 \%$ & 151.0 & 0.986 & 0.922 & 0.095 & 0.105 & $0.00 \%$ \\
\hline \multirow[t]{2}{*}{$b$} & $71.1 \%$ & 150.5 & 0.986 & 0.924 & 0.091 & 0.104 & $0.00 \%$ \\
\hline & $71.0 \%$ & 150.0 & 0.983 & 0.925 & 0.082 & 0.106 & $0.00 \%$ \\
\hline \multirow[t]{2}{*}{$\mathrm{c}$} & $70.9 \%$ & 149.5 & 0.979 & 0.924 & 0.074 & 0.103 & $0.00 \%$ \\
\hline & $70.8 \%$ & 149.1 & 0.973 & 0.920 & 0.063 & 0.102 & $0.00 \%$ \\
\hline \multirow[t]{4}{*}{ d } & $70.6 \%$ & 148.5 & 0.966 & 0.914 & 0.052 & 0.101 & $0.00 \%$ \\
\hline & $70.4 \%$ & 148.0 & 0.959 & 0.911 & 0.051 & 0.096 & $0.00 \%$ \\
\hline & $70.2 \%$ & 147.5 & 0.953 & 0.911 & 0.055 & 0.091 & $0.00 \%$ \\
\hline & $70.0 \%$ & 147.1 & 0.948 & 0.910 & 0.056 & 0.092 & $0.00 \%$ \\
\hline
\end{tabular}

a) Cases with no boundary-layer bleed.

\begin{tabular}{|c|c|c|c|c|c|c|c|}
\hline & $70.5 \%$ & 148.7 & 0.978 & 0.934 & 0.110 & 0.094 & $1.00 \%$ \\
\hline & $70.0 \%$ & 147.2 & 0.978 & 0.941 & 0.081 & 0.092 & $1.00 \%$ \\
\hline a & $69.5 \%$ & 146.0 & 0.976 & 0.947 & 0.040 & 0.095 & $1.07 \%$ \\
\hline & $69.0 \%$ & 144.7 & 0.974 & 0.952 & 0.023 & 0.089 & $1.24 \%$ \\
\hline b & $68.5 \%$ & 143.4 & 0.970 & 0.957 & 0.034 & 0.083 & $1.46 \%$ \\
\hline & $68.3 \%$ & 142.8 & 0.967 & 0.954 & 0.043 & 0.079 & $1.66 \%$ \\
\hline c & $68.2 \%$ & 142.5 & 0.963 & 0.954 & 0.038 & 0.072 & $1.79 \%$ \\
\hline d & $68.1 \%$ & 142.2 & 0.953 & 0.946 & 0.020 & 0.070 & $1.93 \%$ \\
\hline & $68.0 \%$ & 142.0 & 0.944 & 0.936 & 0.021 & 0.071 & $1.97 \%$ \\
\hline & $67.5 \%$ & 141.1 & 0.926 & 0.928 & 0.021 & 0.067 & $2.00 \%$ \\
\hline
\end{tabular}

b) Cases with boundary-layer bleed. 
critical, and "c" and "d" are sub-critical. These cases will be examined in more detail. Note that the variation in nozzle throat area required to resolve performance around the critical point was as little as one-tenth of one-percent. The inlet capture area was the same for the baseline and bleed cases. In practice, the capture area of the bleed case would be larger to maintain the design corrected air flow at the engine face.

Figure 21 presents Mach number contours on the symmetry plane and total pressure contours at the AIP for the four cases with no bleed highlighted in Table 1. The white dashed line denotes the terminal shock in the analytical parent flowfield for comparison to that at the critical point (case "b") and as a reference in the other cases. At the critical point, the terminal shock is skewed somewhat by spillage through the increased vent area. As back-pressure increases from cases "a" to "d" the terminal shock tends to align itself with a conical ray along which Mach number and pressure are constant. However, at the higher back pressures, the terminal shock is increasingly misaligned with the conical rays resulting in the transverse gradients seen in the subsonic diffuser. Lastly, the terminal shock boundarylayer interaction becomes more pronounced as back-pressure and the local Mach number increase. Total pressure contours at the AIP appear on the right side of Fig. 21. Area-weighted probe locations used to evaluate performance parameters are also shown. A substantial region of low-momentum flow develops on the upper surface of the subsonic diffuser in all cases. The extent of this low-momentum region is maximum at $0^{0}$ (top dead center), and minimum at $180^{\circ}$, consistent with the length of boundary-layer run upstream of the terminal shock. The severity of the momentum deficit decreases as back pressure is increased, in opposition to increasing severity of the terminal shock boundarylayer interaction. Therefore, the shock interaction is not a primary contributor to the momentum deficit at the AIP and boundary-layer treatment such as bleed or vortex generators at the subsonic diffuser entrance should be effective.

The effect of boundary-layer bleed is shown in Fig. 22. Bleed flow increases with back-pressure as seen in Table 1 , and was $1.46 \%$ for case " $\mathrm{b}$ " at the critical point. Most notable is a reduction in the extent of the low-momentum

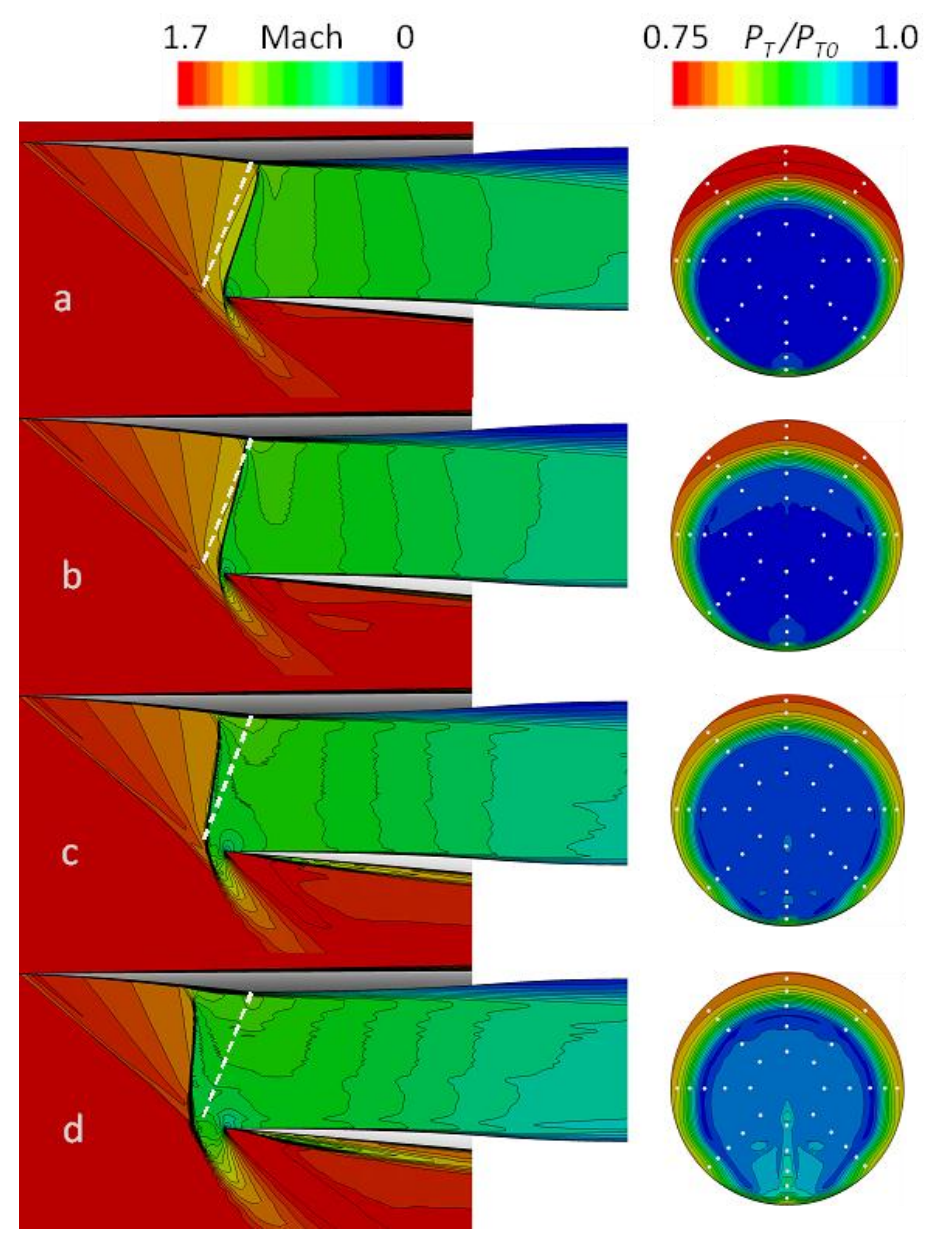

Figure 21. Mach number contours on the symmetry plane and total pressure recovery contours at the AIP for the cases with no boundary-layer bleed. 
region at the AIP. There is also evidence of a vortical structure roughly $45^{0}$ from due to flow misalignment at the swept edges of the modified vent region. For this preliminary design, no attempt was made to optimize the vent region geometry, nor the bleed pattern and rate. The use of vortex generators at the subsonic diffuser inlet was also not explored and may be a viable alternative to bleed.

Figures 23 and 24 summarize inlet performance with, and without bleed in terms of total pressure recovery and

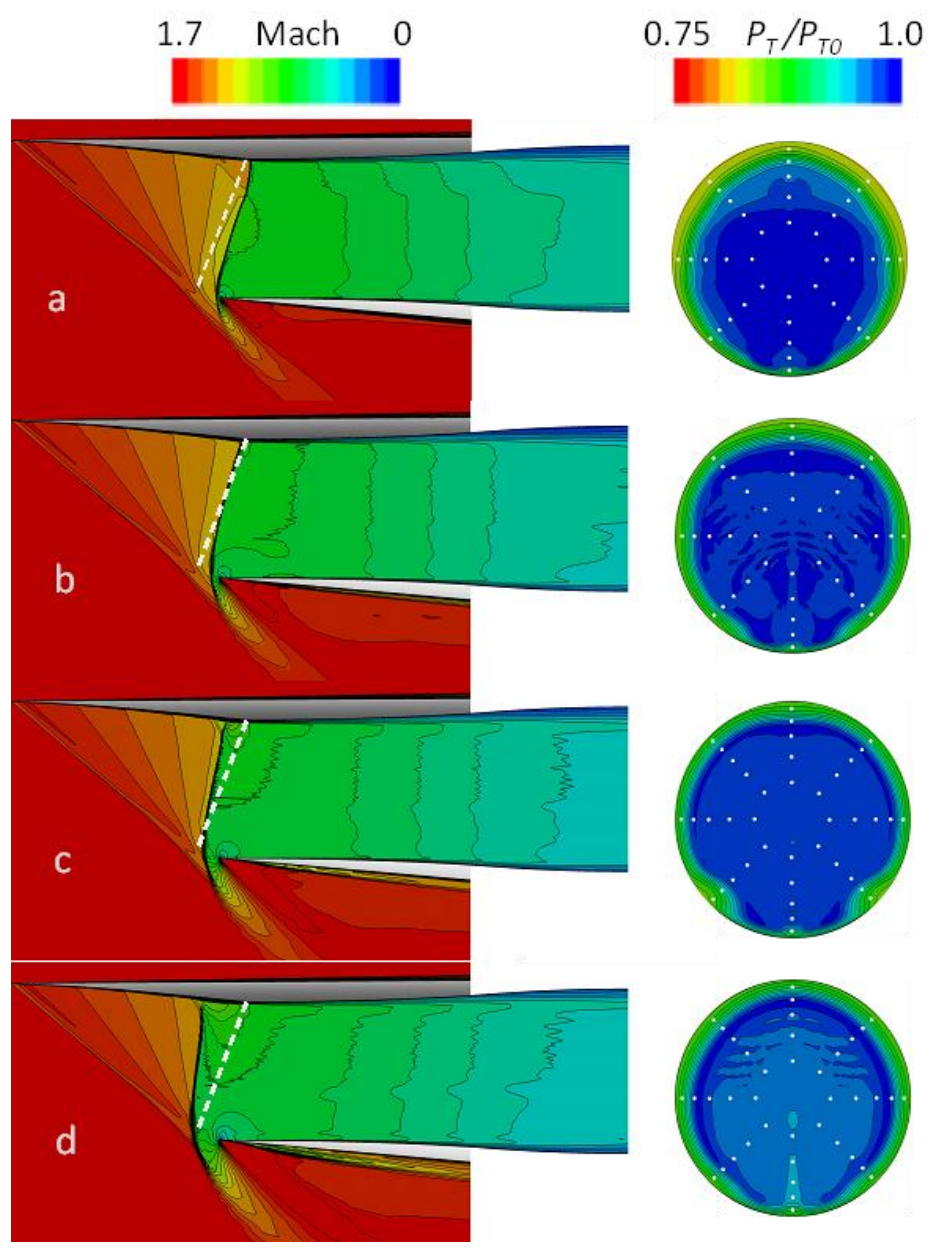

Figure 22. Mach number contours on the symmetry plane and total pressure recovery contours at the AIP for the cases with boundary-layer bleed.

distortion. Figure 23 shows that the effect of bleed is to increase total pressure recovery by $3.5 \%$ at the critical point to just above the MIL-E-5007D value for Mach 1.7. The difference in mass flow ratio due to bleed is evident, as well as a $1.3 \%$ deficit in mass flow ratio for the no-bleed case at supercritical conditions due to supersonic spillage at the enlarged vent region. Recalling the 0.989 total pressure recovery of the parent flowfield, the additional loss of roughly $3 \%$ is attributed primarily to viscous losses in the subsonic diffuser. 


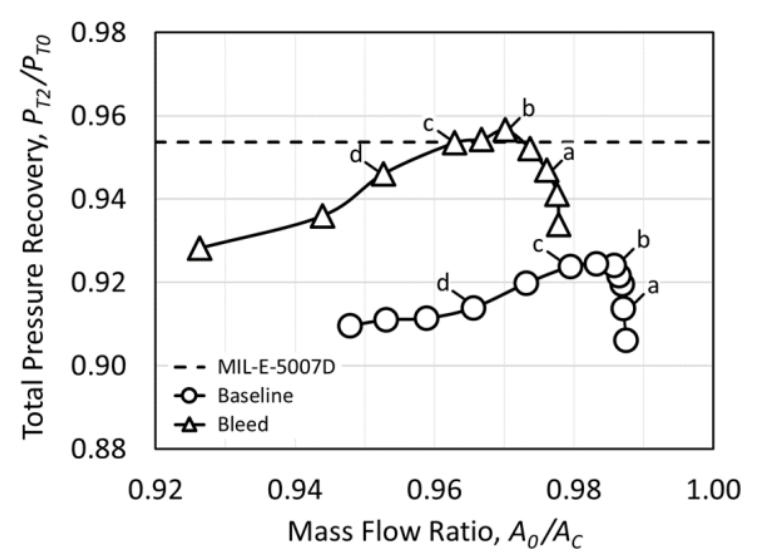

Figure 23. Effect of boundary-layer bleed on inlet total pressure recovery characteristics.

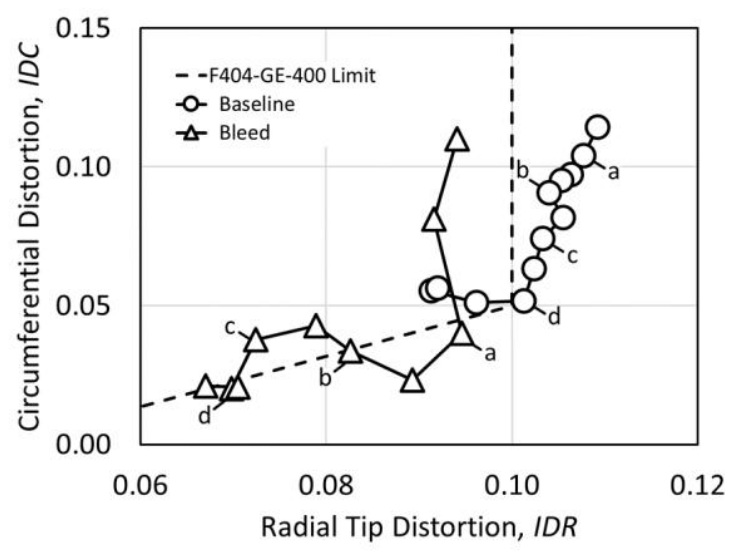

Figure 24. Effect of boundary-layer bleed on inlet distortion characteristics.

Figure 24 presents distortion for the bleed and no-bleed cases in terms of the distortion parameters of reference 17. The design limits for the F404-GE-400 turbofan engine from reference 18 are also shown. In general, both radial and circumferential distortion decrease as back-pressure increases and the inlet transitions from super- to sub-critical operation. Boundary-layer bleed significantly reduced both radial and circumferential distortion parameter values at a given operation condition. At the critical point (b), radial tip and circumferential distortion were reduced by $2 \%$ and $5 \%$ respectively. Further reduction in radial tip distortion is required to provide adequate margin for off-design, installation, and angle-of-incidence effects.

\section{Summary and Conclusions}

The present work has improved the internal performance of streamline-traced, inward-turning inlets in order that the sonic boom and drag advantages shown by previous authors can be realized. This was done by prudent design of the parent flowfield and application of a new technique for merging internal conical flow "A" (ICFA) and Busemann flowfields.

The ICFA flowfield was used to incorporate the inward-turning cowl leading edge for low drag and sonic boom. This was merged with a Busemann conical compression, terminating in a strong oblique wave. A three-shock architecture was also considered, and may be useful for higher flight Mach number applications. A number of merging techniques were assessed using axisymmetric Euler CFD analysis. The best result was obtained by expanding the ICFA exit Mach number about the difference in flow deflection angles at the ICFA exit ray, and matching that to the Busemann inlet Mach number. A study of basic design parameters revealed that a 5-degree inward cowl leading edge angle and an outflow Mach number of 0.9 were appropriate choices for the present application to Mach 1.7 flight conditions. Mach 2 is a practical limit for the two-shock architecture if a terminal shock Mach number of 1.3 or less is to be maintained.

A Mach 1.7 streamline-traced inlet was developed based on the two-shock parent flowfield architecture. Modifications to the native geometry included rounding of the sharp corner at the exit shock impingement point, relieving the flow surface to accommodate the boundary-layer displacement thickness, and enlargement of the vent region near the focal point of the parent flowfield. Three-dimensional Reynolds-Averaged Navier-Stokes CFD simulations were conducted on the resulting geometry over a range of corrected flow rates with and without boundarylayer bleed in the vicinity of the exit shock wave. At the inlet critical point, $1.46 \%$ bleed flow increased total pressure recovery by $3.5 \%$ to $95.7 \%$. In general, distortion decreased with increasing back-pressure, and was also reduced substantially by boundary-layer bleed. However, distortion remained near the edge of the F404-GE-4000 operational envelope due to the radial component. Means to reduce the radial distortion include lengthening the subsonic diffuser, optimization of the bleed scheme, and the use of vortex generators. 


\section{Acknowledgements}

The authors wish to acknowledge support from the NASA Commercial Supersonic Technology Project and the Advanced Air Vehicles Program as well as the NASA Aeronautics Research Mission Directorate Aeronautics Scholarship Program.

\section{References}

${ }^{1}$ Molder, S., and Szpiro, E. J., "Busemann Inlet for Hypersonic Speeds," AIAA Journal of Spacecraft and Rockets, Vol. 3, No. 8, August 1966, pp. 1303-1304.

${ }^{2}$ Busemann, A., "Die Achsenssymmetrische Kegelizeuber-Schallstromung,” Luftfahtforschung, Vol. 19, 1942, pp. 137-144.

${ }^{3}$ O'Brien, T. F. and Colville, J. R., "Analytical Computation of Leading-Edge Truncation Effects on Inviscid Busemann-Inlet Performance," AIAA Journal of Propulsion and Power, Vol. 24, No. 4, July-August 2008, pp. 655-661.

${ }^{4}$ Zhao, Z., and Song, W., "Effect of Truncation on the Performance of Busemann Inlet," Modern Applied Science, Vol. 3, No. 2, February 2009, pp. 168-171.

${ }_{5}^{5}$ Billig, F. S., Baurle, R. A., Tam, C-J., and Wornom, S. F., "Design and Analysis of Streamline Traced Hypersonic Inlets," AIAA 1999-4974, November 1999.

${ }^{6}$ Yue, L., Xiao, Y., Chen, L., Chang, X., "Design of Base Flow for Streamline-Traced Hypersonic Inlet,” AIAA 2009-7422, October 2009.

${ }^{7}$ Ramasubramanian, V., Lewis, M., and Starkey, R., "Performance of Various Truncation Strategies Employed on Hypersonic Busemann Inlets," AIAA 2009-7249, October 2009.

${ }^{8}$ You, Y., Zhu, C., Guo, J., "Dual Waverider Concept for the Integration of Hypersonic Inward-Turning Inlet and Airframe Forebody," AIAA 2009-7421, October 2009.

${ }^{9}$ Slater, J. W., Davis, D. O., Sanders, B. W., and Weir, L. J., "Role of CFD in the Aerodynamic Design and Analysis of the Parametric Inlet," ISABE-2005-1168, September 2005.

${ }^{10}$ Slater, J. W., "Methodology for the Design of Streamline-Traced External-Compression Supersonic Inlets," AIAA 20143593, July 2014.

${ }^{11}$ Molder, S., "Internal, Axisymmetric, Conical Flow," AIAA Journal, Vol. 5, No. 7, Jul. 1967, pp. 1252-1255.

${ }^{12}$ Rylov, A. I., "On the Impossibility of Regular Reflection of a Steady-State Shock Wave from the Axis of Symmetry," Journal of Applied Mathematics and Mechanics, Vol. 54, Issue 2, 1990, pp. 201-203.

${ }^{13}$ White, F. W., Viscous Fluid Flow, McGraw-Hill, Inc., New York, 1974, pp. 493-494.

${ }^{14}$ Mani, M., Cary, A. and Ramakrishnan, S. V., "A Structured and Hybrid-unstructured Grid Euler and Navier-Stokes Solver for General Geometry," AIAA 2004-0524, January 2004.

${ }^{15}$ Slater, J. W., "Design and Analysis Tool for External-Compression Supersonic Inlets," AIAA Paper 2012-0016, January 2012.

${ }^{16}$ Society of Automotive Engineering (SAE), "Gas Turbine Engine Inlet Flow Distortion Guidelines," Aerospace Recommended Practice (ARP) 1420, Revision B, February 2002.

${ }^{17}$ Moore, M. T., "Distortion Data Analysis," Air Force Aero Propulsion Laboratory contract 33615-72-C-1763 final report, AFAPL-TR-72-111, December, 1972.

${ }^{18}$ Steenken, W. G., Williams, J. G., Yuhas, A. J., and Walsh, K. R., “An Inlet Distortion Assessment During Aircraft Departures at High Angle of Attack for an F/A-18A Aircraft,” NASA TM-104328, 1997. 